\title{
Two-dimensional low-Reynolds number wind tunnel results for airfoil NACA 0018
}

\author{
W.A. Timmer \\ Wind Energy Section, Faculty of Aerospace Engineering, Delft University of Technology, \\ Kluyverweg I, 2629HS Delft, the Netherlands
}

\begin{abstract}
The two-dimensional characteristics of airfoil NACA 0018 have been measured for Reynolds numbers between $0.15 \times 10^{6}$ and $1.0 \times 10^{6}$ to establish the lift, drag and moment curves that serve as input to performance calculations of vertical axis wind turbines. At the lower surface laminar separation occurs at low to medium angles of attack, which is of significant influence on the characteristics and the radiated noise. For the situation with a lower surface laminar separation bubble, span wise wake rake traverse measurements showed an irregular three-dimensional pattern. Noise reduction could be achieved with zigzag tape at the $70 \%$ to $80 \%$ lower surface chord station. Significant post-stall hysteresis loops occurred showing a high loss in lift.
\end{abstract}

\section{INTRODUCTION.}

In the last decade the rising price of fossil fuels has increased the attention for the application of wind energy in the urban environment, with a focus on the use of vertical axis wind turbines (VAWT). As for their horizontal axis counter parts, the stiffness and strength of the vawtblades are of paramount importance for a successful design.

Generally VAWT's have symmetrical blade profiles and many of the blade designs rely on the old NACA 0018 airfoil as a balance between aerodynamic performance and structural integrity. It appears however that, despite the many applications, information on the performance of this airfoil at the appropriate Reynolds numbers and test conditions is nonexistent, or at the least very limited. This makes it not only difficult to predict turbine parameters like power output and starting wind velocity, but is also hampers the design process of improved airfoils, since it is unclear what to compete with.

The wind tunnel tests described in this paper aimed at generating steady two-dimensional low-Reynolds number characteristics of the NACA 0018 airfoil, in view of creating a base-line as a starting point for new improved airfoil designs.

If we consider a Darrieus-type turbine with twisted blades (Turby, figure 1) having a tip speed ratio of 3 and blade chords of 10 tol $\mathrm{cm}$, the Reynolds numbers typically range between $0.25 \times 10^{6}$ and $0.5 \times 10^{6}$. For the present tests the Reynolds number range has been chosen sufficiently large, between $0.15 \times 10^{6}$ and $1.0 \times 10^{6}$. 


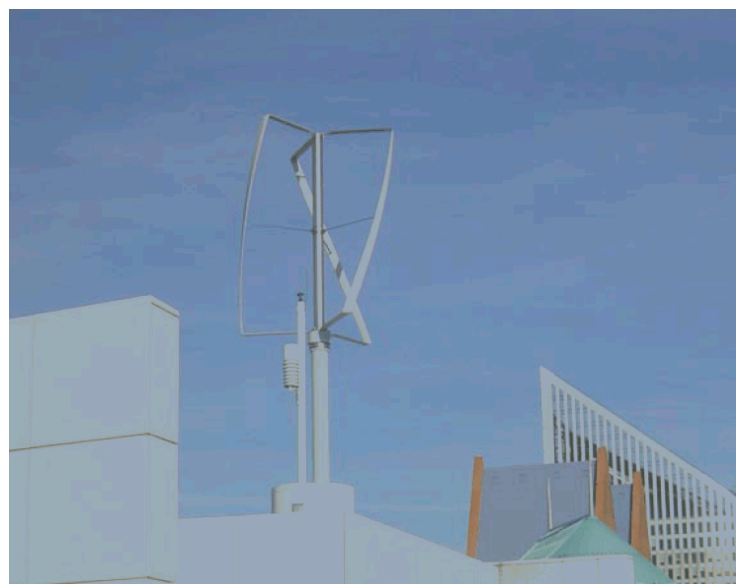

Figure 1: Example of a VAWT [1].

\section{TEST SETUP.}

The measurements were performed in the Delft University Low-speed Wind Tunnel. A schematic of the tunnel layout is depicted in figure 2 . The maximum wind speed is $120 \mathrm{~m} / \mathrm{s}$ and the turbulence intensity in the test section varies from $.02 \%$ at $10 \mathrm{~m} / \mathrm{s}$ to $.07 \%$ at $70 \mathrm{~m} / \mathrm{s}$ corresponding to Reynolds numbers in the range from about $0.15 \times 10^{6}$ to $1 \times 10^{6}$ using $0.25 \mathrm{~m}$ chord models.

The forces and moments on the model were recorded with a six component mechanical balance system and the drag was also measured with a wake rake consisting of 50 total and 12 static pressure tubes.

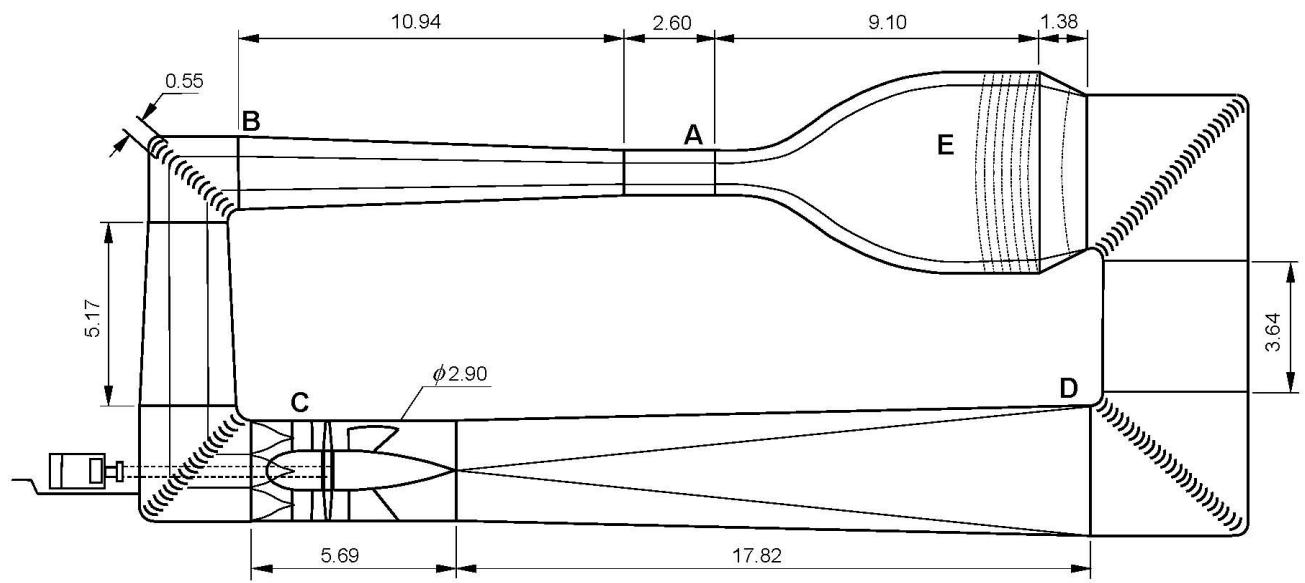

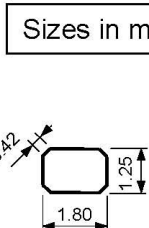

A

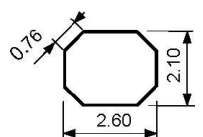

B

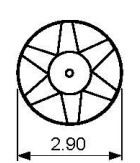

C

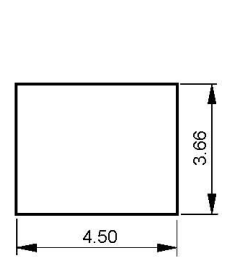

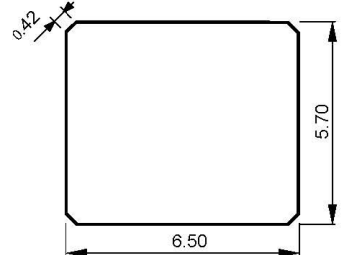

E

Figure 2: A schematic of the Delft low-speed wind tunnel layout 
The $0.25 \mathrm{~m}$ chord numerically milled steel model was placed horizontally in the tunnel, completely spanning the $1.80 \mathrm{~m}$ width of the test section, but leaving a gap at both ends smaller than $0.5 \mathrm{~mm}$. Figure 3 gives an overview of the model in the test section.

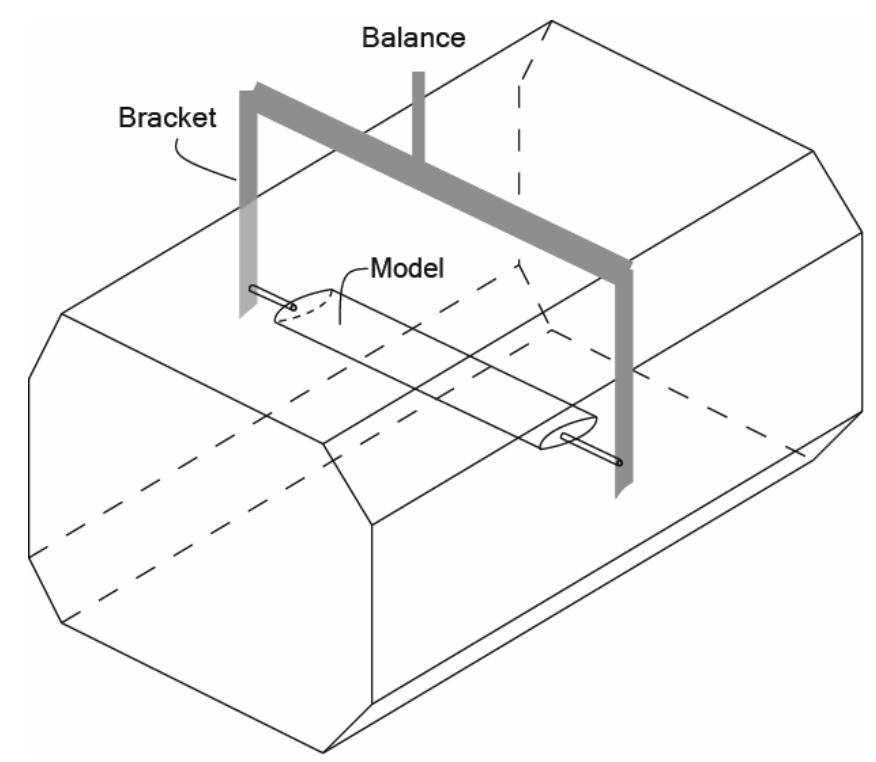

Figure 3: The model attached to the balance system by means of a bracket.

The pressures in the wake were recorded with a liquid manometer. The liquid level was read by fiber-optic cells with an accuracy of $2 \mathrm{~N} / \mathrm{m}^{2}$.

\section{PREDICTION OF CHARACTERISTICS}

\section{I Natural transition}

Calculation were performed with RFOIL [2], a modified version of the well known XFOIL code developed by Drela at MIT, using non-linear amplification of the boundary-layer. Figures 4 and 5 depict results of the calculations at a Reynolds number of $0.7 \times 10^{6}$.
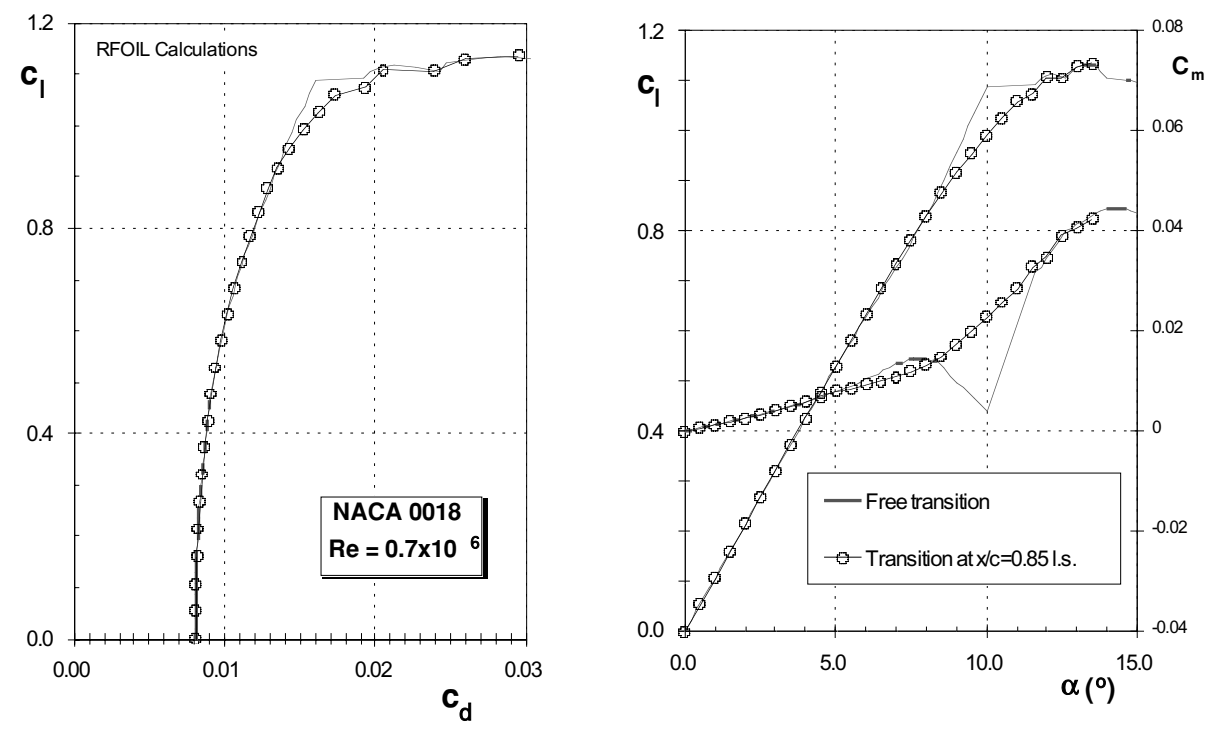

Figure 4: RF0IL Calculated characteristics of NACA 0018 at $\mathrm{Re}=700,000$. Non-linear amplification scheme. L.s.=lower surface 
Despite the relatively low Reynolds number, the calculations indicate that upper surface laminar separation bubbles are not formed until 8 degrees. Although the values of the friction force coefficient $\mathrm{C}_{\mathrm{f}}$ are very small at smaller angles of attack, they do not drop below zero (when it is assumed laminar separation takes place) prior to 8 degrees. At 0 degrees transition takes place at $\mathrm{x} / \mathrm{c}=54 \%$. With increasing angle of attack transition moves forward on the upper surface until at 8 degrees laminar separation starts. At the lower surface transition shifts towards the trailing edge until at 6.5 degrees laminar separation takes place at $\mathrm{x} / \mathrm{c}=92 \%$. With increasing angle of attack this lower surface separation bubble increases in length and stretches towards the trailing edge. The presence of the bubble near the lower surface trailing edge changes the flow conditions in such a way that the outer flow effectively sees a thicker trailing edge, which increases the camber and makes the lift grow stronger than would be the case with ordinary transition somewhere upstream. The increased aft camber reduces the moment coefficient, as can be observed in figure 4 .

At an angle of 10 degrees transition is at the trailing edge. When the angle is increased further, the laminar separation bubble moves further downstream. It extends over the trailing edge, while transition takes place in the wake.

At this point the code has difficulty in converging to a satisfying solution.

At 11.6 degrees the entire lower surface has become laminar. At this angle the flow conditions have changed to normal again and the increase in camber of the airfoil has disappeared. This accounts for the kink in the lift curve and the rapid growth of the moment coefficient.

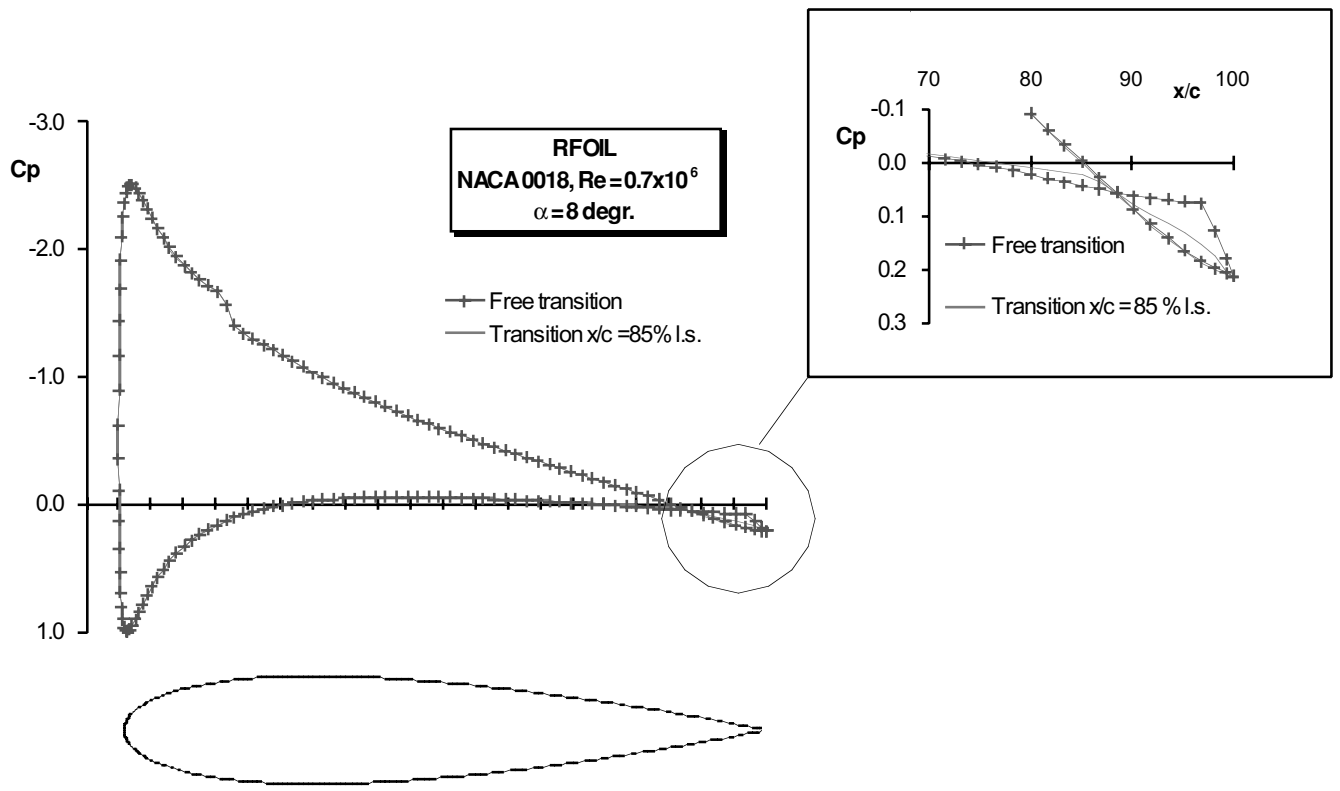

Figure 5: The calculated pressure distribution for NACA 0018 at $\operatorname{Re}=0.7 \times 10^{6}$ and $\alpha=8^{0}$.

\subsection{Forced transition}

As sketched in chapter 3.1 the kink in the lift curve is due to the presence of a lower surface laminar separation bubble with the separation location near $90 \%$ of the chord. Also depicted in figures 4 and 5 is the result of a calculation with forced transition at the lower surface $85 \%$ chord station. It is clear that without the bubble the lift and moment curves show the expected continuous behavior. 


\section{DATA REDUCTION.}

\section{I Wind tunnel wall corrections.}

To the measured data the standard wind tunnel wall corrections have been applied as given in [3]. For the higher angles of attack, in cases of leading edge flow separation, the method of Hackett [4] has been used. At a Reynolds number of 700,000 corrections typically range from $2.5 \%$ in $\mathrm{C}_{\mathrm{l}}$ and $1.7 \%$ in $\mathrm{C}_{\mathrm{d}}$ at 15 degrees angle of attack to $9 \%$ and $8 \%$ respectively at 30 degrees.

\subsection{Induced drag}

The presence of small gaps at both ends of the model, in this case necessary to be able to use the balance, practically turns the two-dimensional model into a large aspect ratio wing. Consequently, data reduction can be performed on the basis of wing theory.

The total drag of a finite wing is given by:

$$
C_{D}=C_{d}+\frac{C_{L}^{2}}{\pi e A R}
$$

where AR is the aspect ratio of the wing, $\mathrm{C}_{\mathrm{d}}$ is the two-dimensional drag coefficient of the wing airfoil and e is the span efficiency (Oswald) factor. For an elliptic wing $\mathrm{e}=1$.

The drag measured by the balance is a combination of induced drag $\left(=0\right.$ at $\left.\mathrm{C}_{\mathrm{l}}=0\right)$ and the interference or parasitic drag $\mathrm{C}_{\mathrm{Dp}}$ caused by the interaction of the flow over the wing and the test section walls boundary layers.

If we take the wake rake drag coefficient to be the two-dimensional drag coefficient and the balance drag coefficient to be $\mathrm{C}_{\mathrm{Db}}$ we can write:

$$
C_{D_{b}}-C_{D_{p}}=C_{d_{w}}+\frac{C_{L}^{2}}{\pi e A R}
$$

If we plot $\Delta C_{D}=C_{D b}-C_{d w}$ versus $C_{L}^{2}$, which is essentially a straight line, we can find the effective model aspect ratio eAR being $\pi$ times the slope of the line and the parasitic drag coefficient at the intercept of the $C_{D}$ axis. In figure 6 this is done for the present measurements at a Reynolds number of $0.7 \times 10^{6}$.

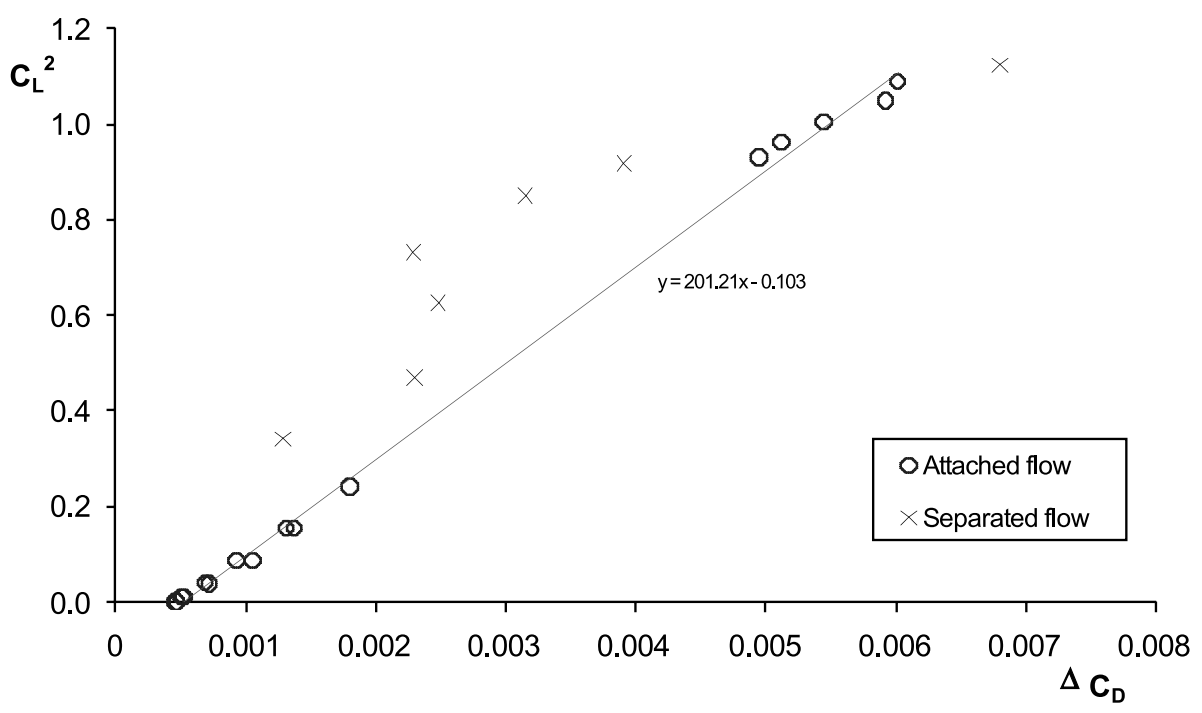

Figure 6: Plot of the induced drag coefficient to find the parasitic drag coefficient and the effective model aspect ratio at a Reynolds number of $0.7 \times 10^{6}$. 
The crosses in figure 6 originate from flow situations where predominantly separation of the boundary layer is present, either laminar separation on the lower surface, or turbulent separation on the upper surface. In these cases the wake shows an irregular 3D-pattern along the span. The wake rake is located at a fixed span position and in situations of significant flow separation generally does not record the average drag of the model. This will be further explored in the next section.

From the plot in figure 6 we can derive the parasitic drag $C_{D p}=0.000512$ and the effective model aspect ratio $e A R=64.06(=201.21 / \pi)$

\section{EXPERIMENTAL RESULTS}

\section{I Characteristics}

Using the method from chapter 4 , the characteristics at the Reynolds numbers $0.3 \times 10^{6}, 0.5 \times 10^{6}$, $0,7 \times 10^{6}$ and $1.0 \times 10^{6}$ were derived. At $\mathrm{Re}=0.15 \times 10^{6}$ the pressures were too low to conduct wake measurements. In this case the drag curve was based on the data for parasitic drag and aspect ratio derived for $\mathrm{Re}=0.3 \times 10^{6}$.
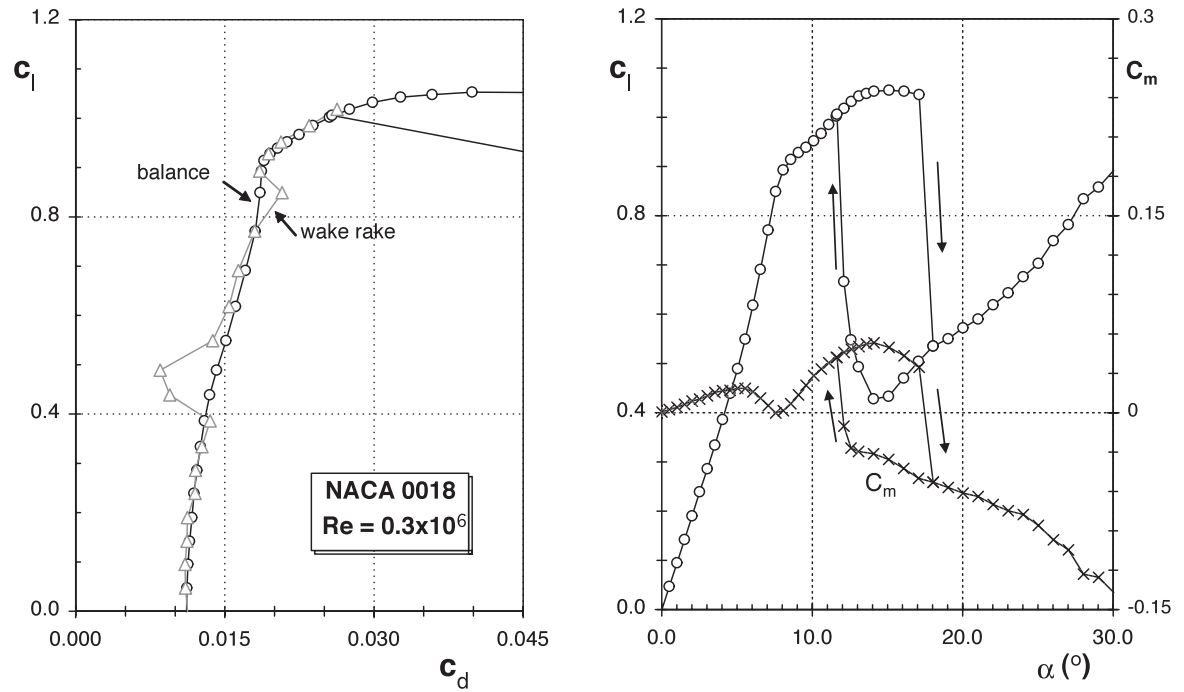

Figure 7a: The measured two-dimensional characteristics of NACA 0018 at $\mathrm{Re}=0.3 \times 10^{6}$.
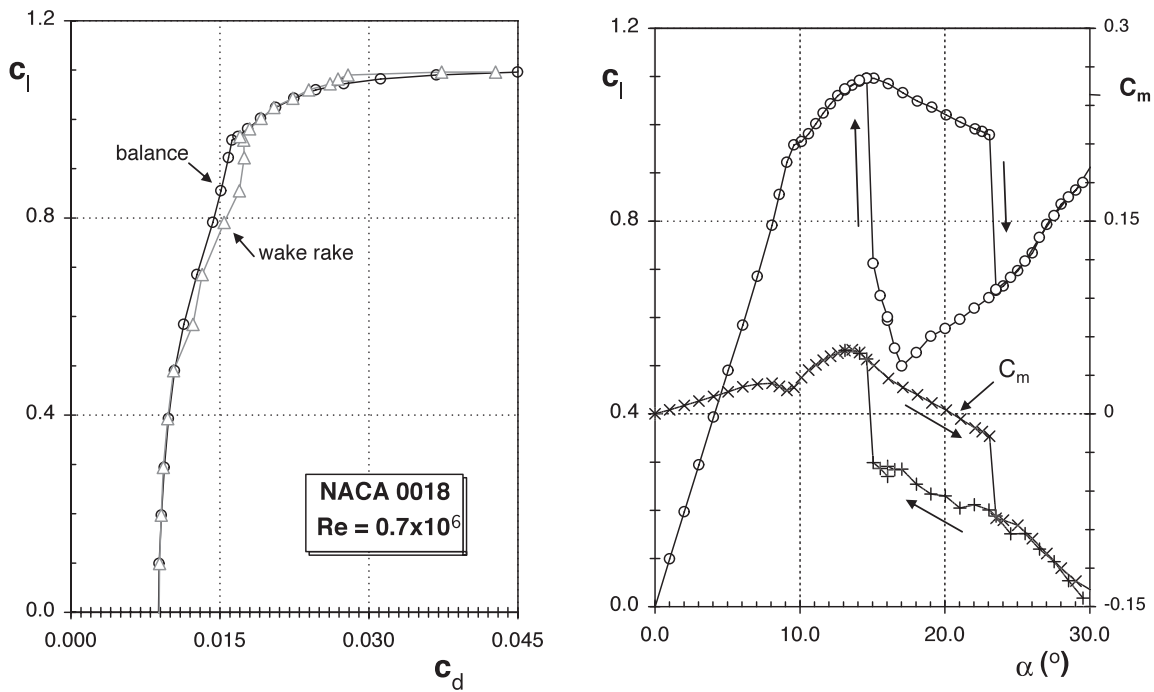

Figure 7b: The measured two-dimensional characteristics of NACA 0018 at $\mathrm{Re}=0.7 \times 10^{6}$. 
Figure 7 shows the performance of NACA0018 at $\mathrm{Re}=0.3 \times 10^{6}$ and $0.7 \times 10^{6}$ demonstrating the effect on the drag curve of the procedure mentioned above.

The figures depict a large deep stall hysteresis loop with a minimum lift coefficient of as low as 0.43 for the lower Reynolds number. The maximum lift coefficients are $1.055\left(0.3 \times 10^{6}\right)$ and 1.096 both at 15.1 degrees. Deep stall enters at 18 degrees for $0.3 \times 10^{6}$ and 23.5 degrees at $0.7 \times 10^{6}$. Although less pronounced, the kink in the lift curves around 10 degrees at $\mathrm{Re}=0.7 \times 10^{6}$ shown in the predictions of figure 4 is also visible here.

\section{I Wake rake traverses}

To check the two-dimensionality of the flow and to find a wake rake span position giving a representative drag for the model, wake rake traverse measurements were performed. In figure 8 a traverse is shown at 8 degrees angle of attack at a Reynolds number of $0.7 \times 10^{6}$. This angle is in the range of angles of attack in which laminar separation bubbles at the lower surface dominate the flow conditions at the trailing edge.

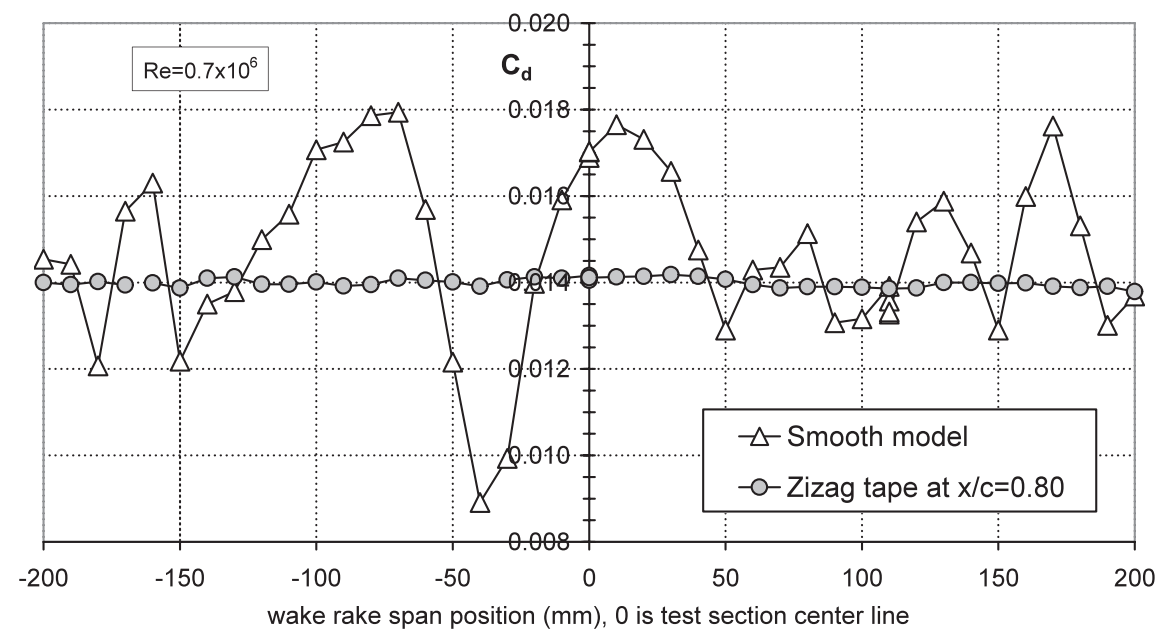

Figure 8: Wake rake traverse measurements at 8 degrees angle of attack showing the effect on span wise wake rake drag of a lower surface laminar separation bubble near the trailing edge.

Clearly, the figure demonstrates the strong three-dimensional character of the flow due to the presence of the lower surface laminar separation bubble. To show that the trouble is indeed caused by the lower surface laminar separation, $0.48 \mathrm{~mm}$ thick zigzag tape was applied just before the lower surface $80 \%$ chord station. For this configuration figure 8 shows a regular wake rake drag pattern along the span.

\subsection{Noise}

The lower surface separation bubble is also responsible for tonal noise, generated at the trailing edge. The flow parameters, such as turbulence intensity, Reynolds stress and nearwall velocities and the noise characteristics for a Reynolds number of 160,000 have been the subject of [5]. In the present measurements the relatively sharp whistling sound disappeared when the boundary layer was tripped. In contrast to the calculations, stethoscope measurements (see figure 9 ) indicate that at $\mathrm{Re}=0.7 \times 10^{6}$ the lower surface laminar separation bubble already stretches across the trailing edge after 6 degrees angle of attack. At this angle backflow in the wake starts to interfere with the upper surface boundary layer and noise is generated. The zigzag trip proved to be an adequate means of reducing the noise. 


\subsection{Measurement of transition}

For Reynolds numbers of $0.5 \times 10^{6}, 0.7 \times 10^{6}$ and $1.0 \times 10^{6}$ the transition location of the boundary layers on the upper and lower surface was measured, using a stethoscope consisting of a small microphone behind a $12 \mathrm{~cm}$ long tube with a diameter of $1 \mathrm{~mm}$. Lines with $5 \%$ chord spacing were drawn on the model to attribute the chord location to a detected transition point.

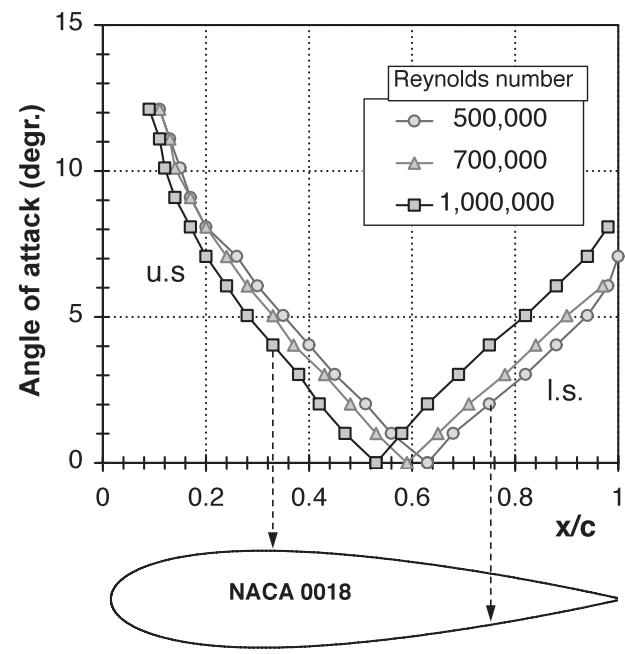

Figure 9a: The transition locations on upper and lower surface for three Reynolds numbers measured with a stethoscope.

Comparisons with infrared measurements and pressure distributions have shown that the accuracy of this method is about 0.5 to $1 \%$ chord. The results are shown in figure $9 a$. The figure demonstrates that for the two lower Reynolds numbers the complete lower surface is laminar at about 7 degrees angle of attack. On the upper surface transition moves steadily forward with increasing angle of attack from $59 \%$ chord at 0 degrees to $11 \%$ chord at 12 degrees for $\mathrm{Re}=0.7 \times 10^{6}$.

Comparison with the calculations in chapter 3 shows that the measured location is approximately $5 \%$ closer to the trailing edge both on the pressure as well as the suction side (figure $9 b$ ).

In cases of transition via a laminar separation bubble it is difficult to judge whether the applied method returns the transition location or that reattachment is detected. In some cases this may lead to a transition location actually projected slightly forward of the one measured with the stethoscope, but this is generally much smaller than $5 \%$.

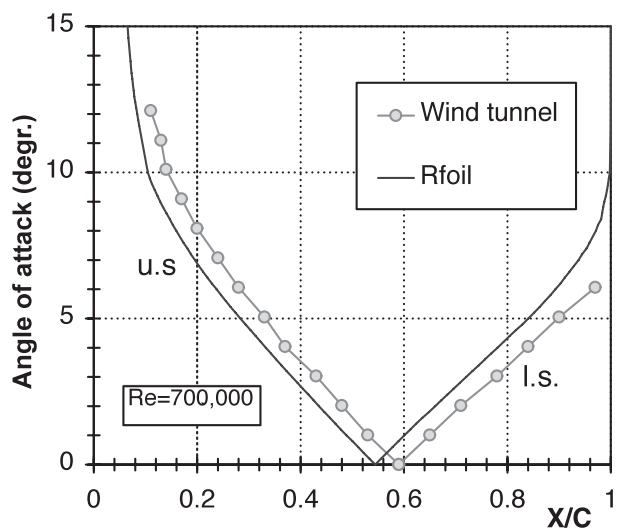

Figure 9b: Comparison of calculated and measured transition locations at a Reynolds number of $0.7 \times 10^{6}$. 
In addition, at the higher angles were the bubbles have disappeared, the difference still is about $5 \%$. No indication, however, exists that the measurements were in error with as much as $5 \%$ chord. The progression of the transition locations on either side of the airfoil with angle of attack seems to be in good agreement with the prediction.

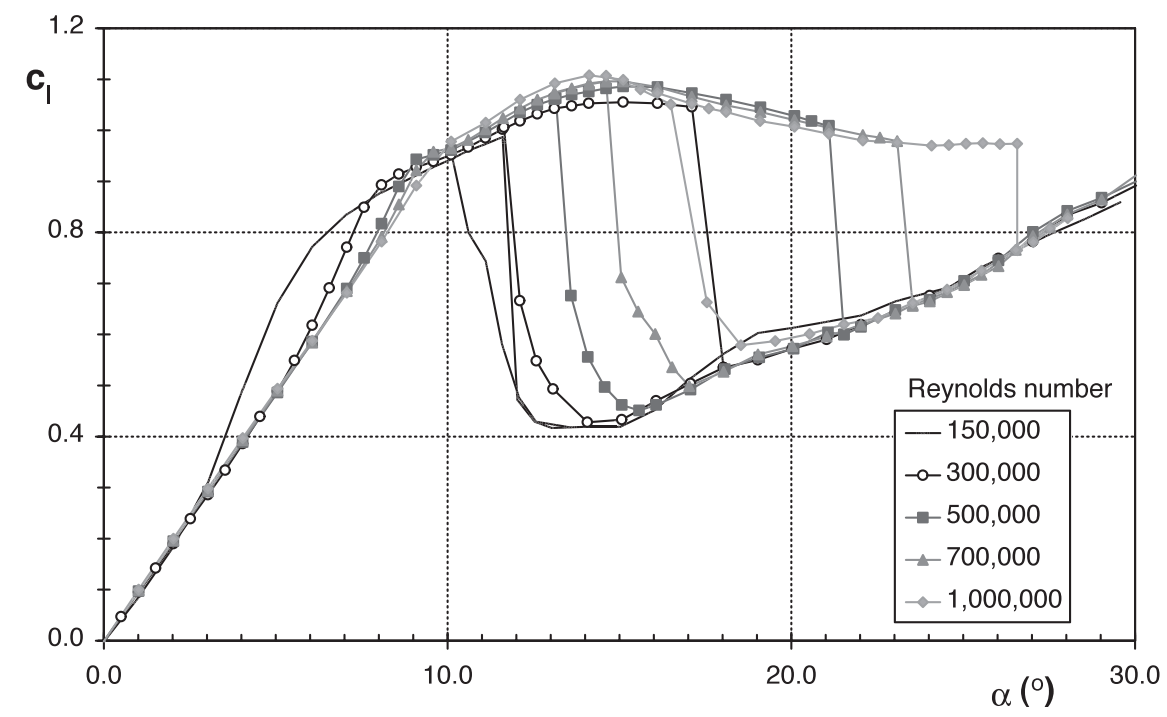

Figure 10a: The effect of Reynolds number on the lift curve of NACA 0018, free transition

\subsection{The effect of Reynolds number with free transition.}

In figure 10 the lift, drag and moment curves are shown with free transition of the boundary layer both on upper and lower surface of the model.

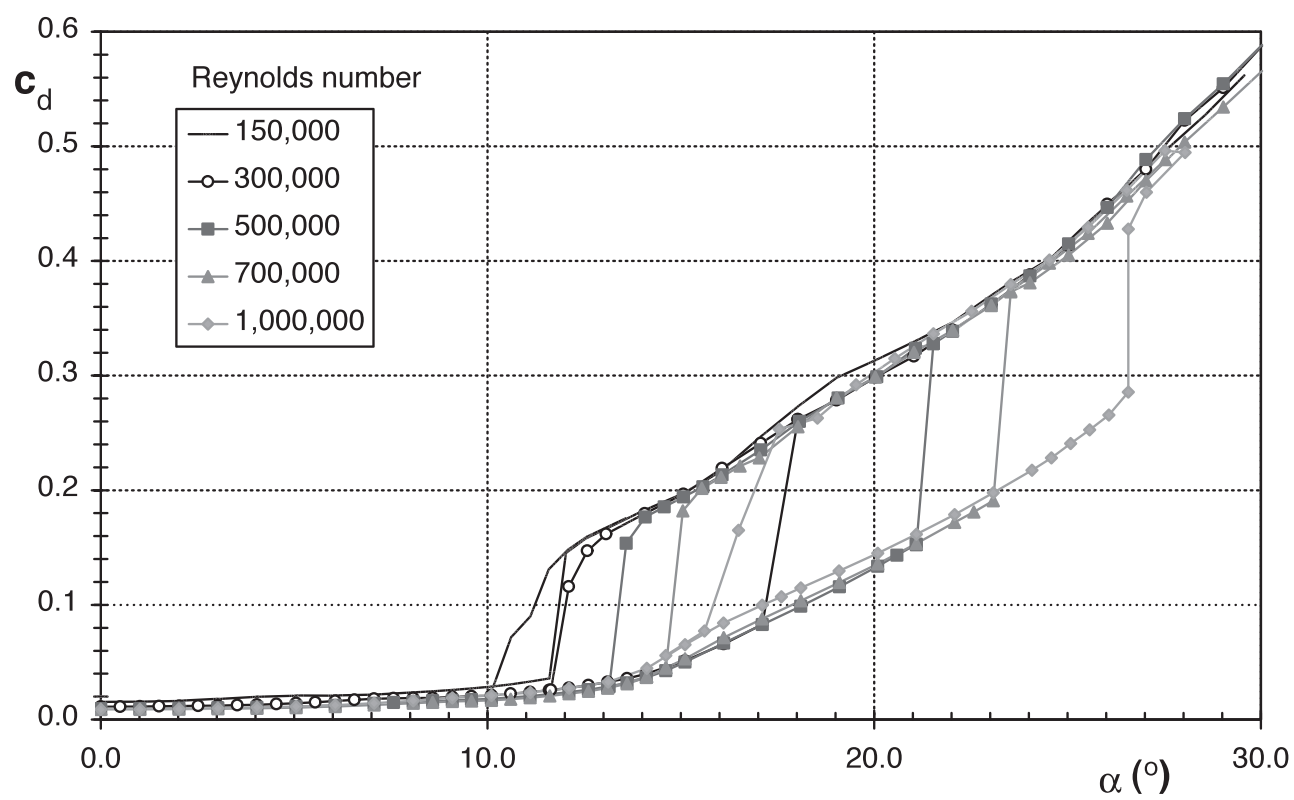

Figure 10b: The effect of Reynolds number on the drag curve of NACA 0018, free transition 


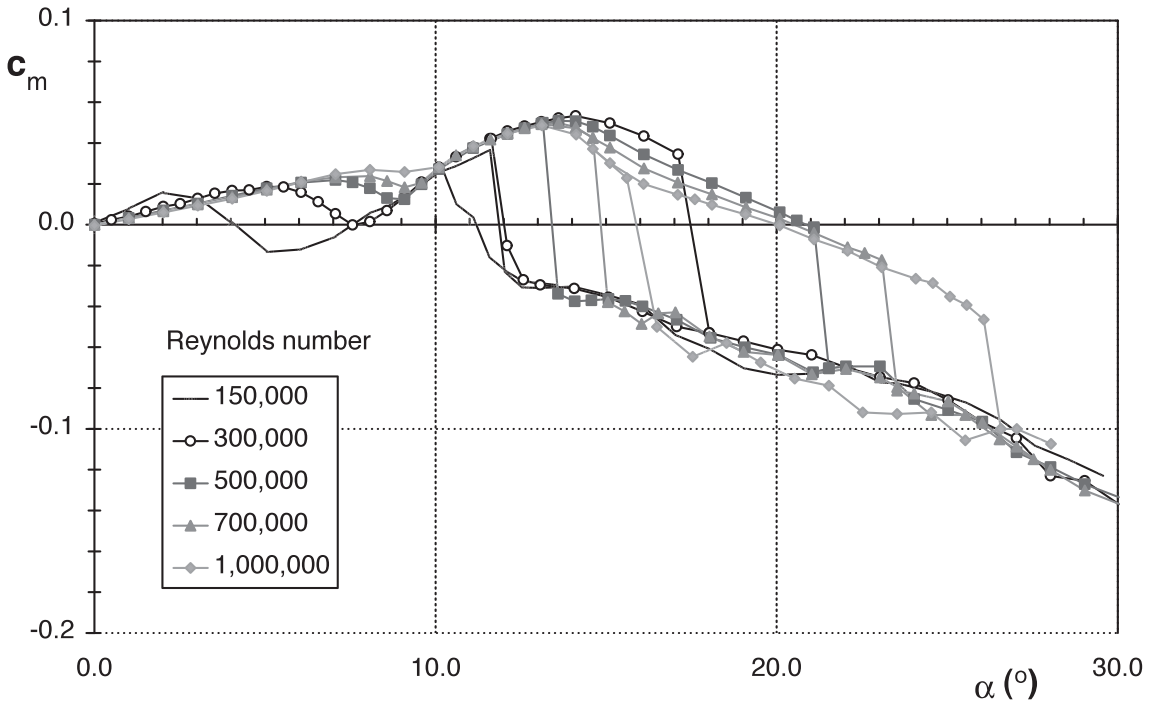

Figure 10c The effect of Reynolds number on the moment curve of NACA 0018, free transition

Some characteristic parameters of the lift and drag curves are tabulated in table 1.

Table I: Airfoil characteristic parameters for NACA 00 I8, free transition

\begin{tabular}{ccccc}
\hline $\operatorname{Re}\left(\mathbf{1 0}^{\mathbf{6}}\right)$ & $\mathbf{C}_{\mathbf{d}, \min }$ & $\mathbf{C}_{\mathbf{l}, \max }$ & $\begin{array}{c}\text { Leading edge } \\
\text { stall angle (degr.) }\end{array}$ & $\begin{array}{c}\text { Length of stall } \\
\text { hysteresis loop (degr.) }\end{array}$ \\
.15 & .01542 & 0.987 & 12.1 & 2.1 \\
.30 & .01173 & 1.055 & 18.0 & 6.4 \\
.50 & .00942 & 1.086 & 21.5 & 7.6 \\
.70 & .00882 & 1.096 & 23.5 & 8.9 \\
1.0 & .00766 & 1.108 & 27.0 & 10.9
\end{tabular}

The tests show an increasing length of the stall hysteresis loop with increasing Reynolds number. Striking is the large loss in lift with decreasing angle of attack which may be attributed to the fairly thick airfoil leading edge. The lift and moment characteristics show a decreasing influence of the lower surface separation bubble in the range of angles of attack between 0 and 10 degrees.
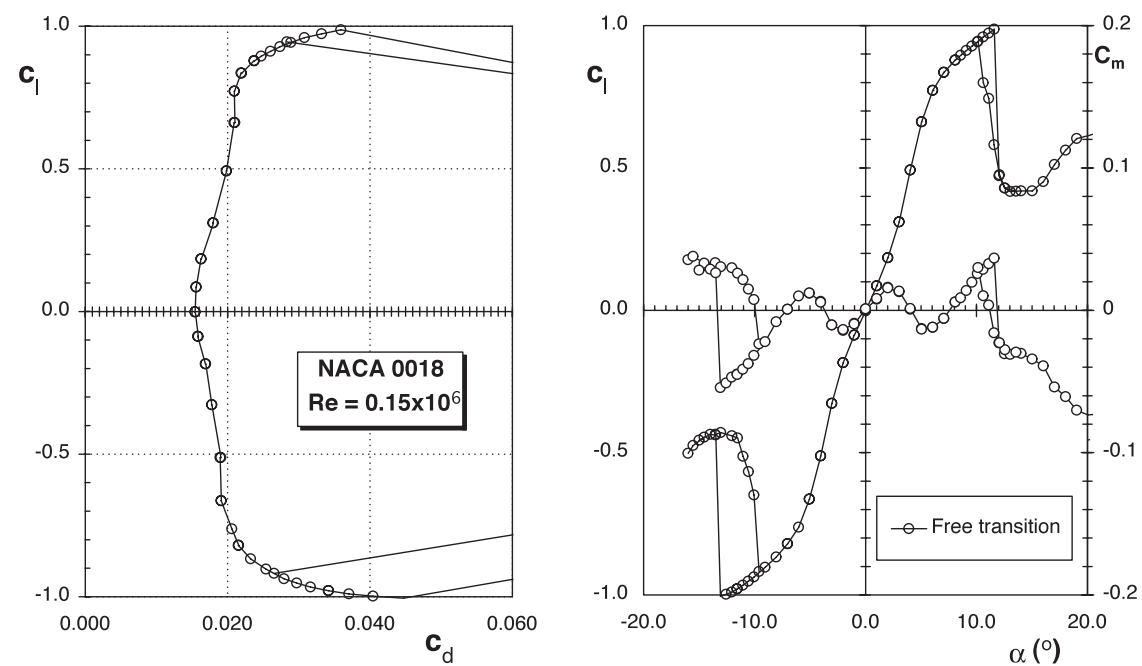

Figure 11: The characteristics of airfoil NACA 0018 at a Reynolds number of 150,000 .

Free transition. 


\subsection{Symmetry}

Since we have a symmetric airfoil, by definition the characteristics at positive angles of attack should match the curves at negative angles. In some publications on symmetrical airfoils such as NACA 0012 and 0015 this aspect received too little attention. In the present tests the balance measurements at positive angles showed excellent agreement with those at negative angles, with the exclusion of the values at the lowest Reynolds number of 150,000 given in figure 11.

The wake rake drag occasionally differed. In those cases a mean value was used for the evaluation of the parasitic drag and the effective model aspect ratio. Furthermore the decreasing angle of attack was corrected for a hysteresis in the angle of attack mechanism of 0.04 degrees.

\subsection{Removing the lower surface laminar separation bubble}

Figure 12 demonstrates the effect on the airfoil performance when at both the upper and lower surface $0.48 \mathrm{~mm}$ thick zigzag tape is located just before the $80 \%$ chord station.
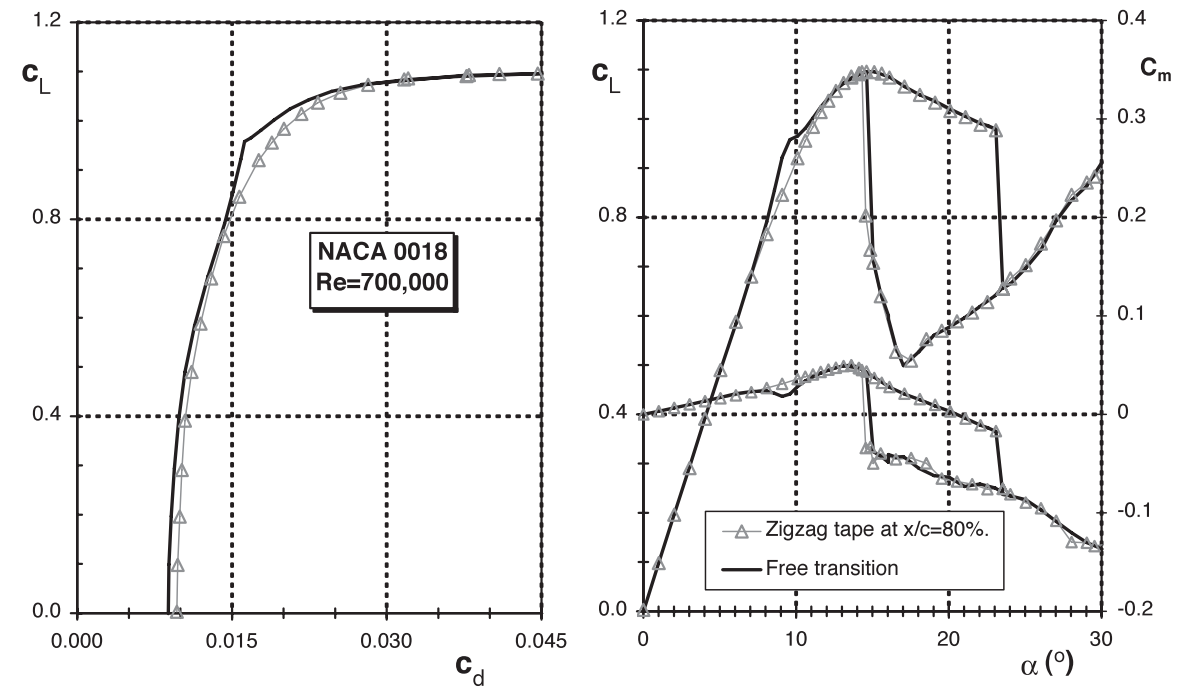

Figure 12: The effect on airfoil performance of fixed transition at the $80 \%$ upper and lower surface chord stations, $\operatorname{Re}=0.7 \times 10^{6}$.

It is clear that the lower surface bubble has disappeared. The tape causes premature transition at the lower surface and the tape thickness adds to the turbulent boundary layer thickness on the upper surface since transition here takes place further upstream. This leads to an increase in the drag coefficient of about 0.0008 for the lower angles of attack

Apart from the kink around 10 degrees the zigzag tape did not affect the lift curve. The same behavior could be observed at $\operatorname{Re}=0.3 \times 10^{6}$, albeit that due to the thicker boundary layer the tape had to be shifted to the $70 \%$ chord position to be effective and that the drag penalty was minimal. 

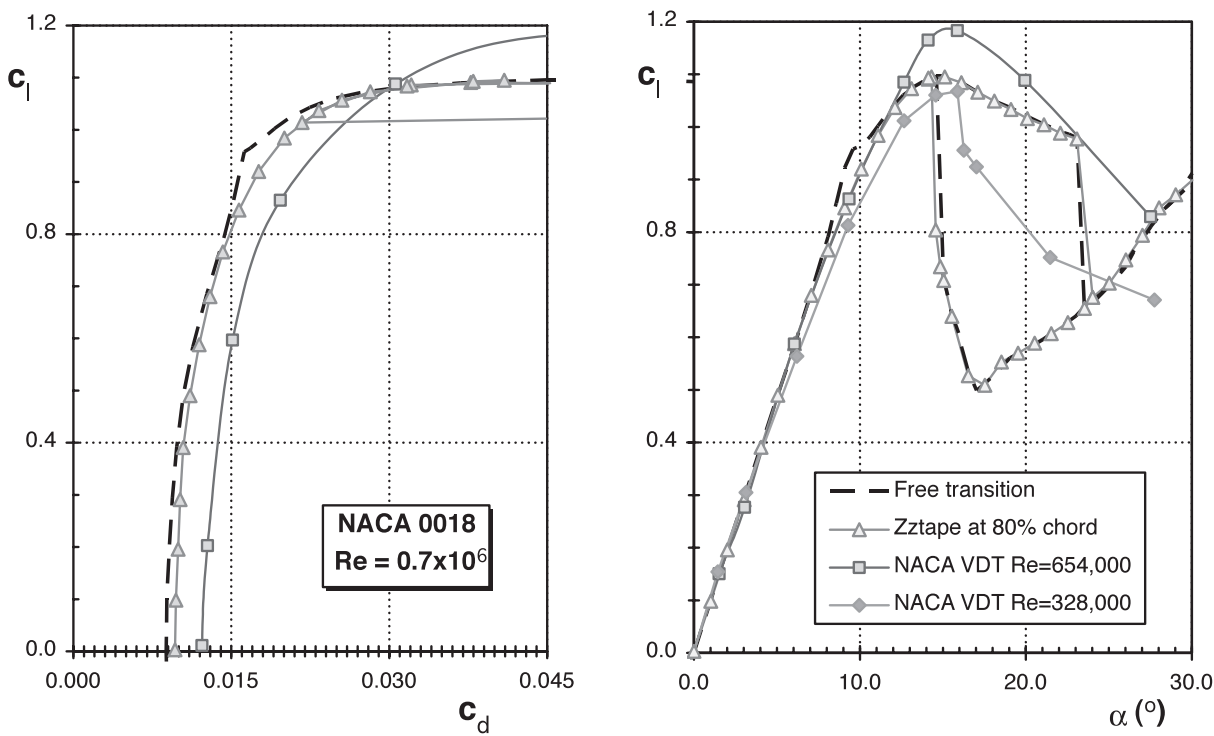

Figure 13: Comparison of the measured characteristics with data from the NACA Variable Density Tunnel [6].

\subsection{Comparison with earlier measurements}

Figure 13 shows a comparison of the present data with those obtained in the NACA variable density tunnel (VDT), [6]. The VDT measurements were already performed in the thirties of the last century. To the author's knowledge no other measurements of this airfoil's characteristics at low to medium Reynolds numbers were reported in literature. The VDT suffered from a high turbulence level, and on basis of the transition location an effective Reynolds number was established being 2.64 times the test Reynolds number. In figure 13 two lift curves from the VDT are shown. The data at a test Reynolds number of $0.65 \times 10^{6}$ and those at an effective Reynolds number of $0.87 \times 10^{6}$ (test Reynolds number $0.33 \times 10^{6}$ ) are compared with the present measurements at $0.7 \times 10^{6}$. Resulting from the increased turbulence the VDT lift data do not show the kink due to the lower surface separation bubble. The lift gradient of the curve at $0.65 \times 10^{6}$ seems to match the present measurements with zigzag tape at both sides to prevent laminar separation. However, the maximum lift coefficient is 0.11 units too high. The effective Reynolds number curve has a maximum lift coefficient comparable to the present tests, but the lift gradient is not steep enough. The post stall data differ significantly, which was to be expected in view of the fact that the VDT coefficients basically were derived from wing tests and consequently inherently have a three-dimensional character.

The VDT drag curve matches the zigzag tape tests for the lower angles of attack when a factor of 0.8 is applied.

Figure 14 presents the effect of the Reynolds number on the maximum lift coefficient for the present tests and for the NACA VDT.

The figure shows that the present measurements form a sort of cross-over between the VDT curves for test Reynolds number and effective Reynolds number since, with increasing Reynolds number, the transition location in the present tests comes closer to the one defined for the VDT effective Reynolds number. 


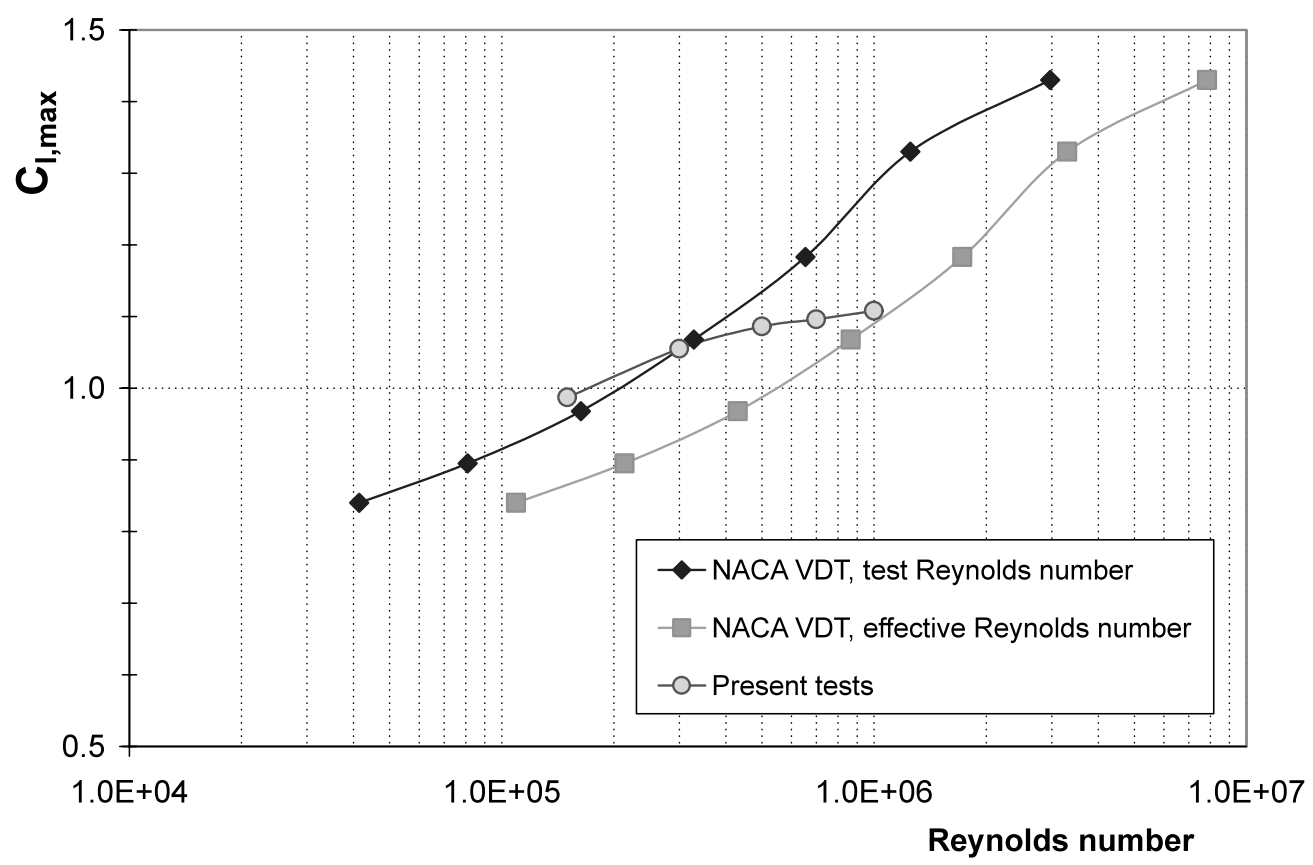

Figure 14: The effect of Reynolds number on the lift coefficient of NACA 0018, free transition. Note that the vertical axis starts at 0.5

\section{CONCLUSION}

Balance and wake rake measurements were performed on a $0.25 \mathrm{~m}$ chord model having a NACA 0018 airfoil in the Delft University low-turbulence wind tunnel. The tests show that a lower surface laminar separation bubble dominates the flow and noise characteristics at Reynolds numbers between $0.15 \times 10^{6}$ and $1.0 \times 10^{6}$. When the lower surface bubble was removed by tripping the boundary layer with zigzag tape the tonal noise disappeared. Large post stall hysteresis loops are present which increase in length with increasing Reynolds numbers, while the leading edge separation angle of attack shifts to higher values. The measured transition locations are about $5 \%$ chord behind the calculated values, however the effect of angle of attack on transition seems to match the predictions quite well.

\section{REFERENCES}

1. Website of the Turby vertical axis turbine, www.turby.nl

2. R. van Rooij, "Modification of the boundary layer calculation in RFOIL for improved airfoil stall prediction". Report IW-96087R, TU-Delft, the Netherlands

3. H.C. Garner, E.W.E. Rogers, W.E.A. Acum and E.C. Maskell, "Subsonic wind tunnel wall corrections". AGARDograph 109, October 1966

4. $\quad$ B.F.R. Ewald (Ed.), "Wind tunnel wall corrections". AGARDograph 336, October 1998

5. T. Nakano et al. "Experimental study on flow and noise characteristics of NACA 0018 airfoil". Journal of Wind Engineering and Industrial Aerodynamics 95 (2007) pp 511-531

6. Jacobs, Eastman N Sherman, Albert. "Airfoil section characteristics as affected by variations of the Reynolds number”. NACA Report 586, (1937). 
